\title{
Vitamin C Content in Ultra-Violet-C Irradiated Tomatoes
}

\author{
Sucharitha K. V ${ }^{1}$, Beulah A. M $^{2}$ \& Hymavathi $\mathrm{U}^{3}$ \\ ${ }^{1},{ }^{2},{ }^{3}$ Department of Home Science, Sri Venkateswara University Tirupati, A.P, 517502, INDIA
}

\begin{abstract}
The effect of ultra-violet irradiation $(U V-C)$ on vitamin-C content in fresh cut tomatoes was investigated. Fresh tomatoes (Lycopersicon esculentum) were blanched at $60^{\circ} \mathrm{c}$ for 5 min and cut into equal size pieces. Tomatoes of $100 \mathrm{~g}$ (Sample-A) exposed to UV-C of 254nm, $3.6 \mathrm{KJ}$ for 5 minutes, and to sample $B$ 5\% of garlic was added as natural preservative, in addition to UV-C treatment. The sample-C was kept as control without any treatment. The mean vitamin-C content in sample $A$ and $B$ was significantly more than the sample $C$. The shelf life of sample A and B extended up to 45 days without deterioration. So UV energy can be used safely to extend the shelf-life of tomatoes.
\end{abstract}

Keywords: Garlic, Shelf-life, Tomatoes, UV-C Irradiation, vitamin-C

\section{Introduction}

Tomato is the world's largest vegetable crop. The lycopene and vitamin C present are powerful antioxidants helps in preventing the degenerative diseases $[1,2,3]$.The emerging technology UV-C irradiation is a type of non-ionizing radiation.UV-C irradiation at $245 \mathrm{~nm}$ has the highest germicidal action, surface decontamination and the control of microorganisms growth in fresh cut products.[4].Natural antimicrobial compounds are re -emerging alternative to fresh cut tomatoes and its products preservation. Garlic constituents possess broad spectrum of antibiotic effects, antiseptic, antifungal and antimicrobial both internally as well as externally [5]. The present study was to analyze the vitamin- C content of fresh cut tomatoes exposed to UV-C irradiation and natural preservative garlic.

\section{Materials And Methods}

Fresh tomatoes of local breed (Lycopersicon esculentum) with uniform size, shape and ripened fruits were collected from agricultural nursery.

\subsection{Processing of raw materials}

Fresh tomatoes were collected, washed with distilled water and were blanched at $60^{\circ} \mathrm{c}$ for 5 mins and were cut into pieces and filled into pre sterilized containers and stored at $10^{\circ} \mathrm{c}$ in a refrigerator for further study.

\subsection{UV-C irradiation method:-}

A low pressure mercury vapour discharge ( Phillips germicidal sterile) lamp with a tubular glass envelope emitting short wave UV radiation with a peak of $254 \mathrm{~nm}$ (UV-C) with $3.6 \mathrm{~kJ} / \mathrm{m}^{2}$ exposed for $5 \mathrm{~min}$ according to the procedure described by Steven's et al (1998) [6].

\subsection{Preparation of samples:-}

1.3.1 Sample A: Tomatoes of $100 \mathrm{~g}$ were taken into pre sterilized containers were exposed to UV radiation for about 5 minutes and stored at $10^{\circ} \mathrm{c}$ in a refrigerator.

1.3.2 Sample B: - To $100 \mathrm{~g}$ of tomatoes $5 \%(5 \mathrm{~g} / 100 \mathrm{~g})$ of garlic paste was added as natural preservative and exposed to UV light for about $5 \mathrm{~min}$.

1.3.3 Sample C: - Control is a sample of tomatoes without any treatment.

\subsection{Shelf life studies}

All the samples (A, B \& C) were stored in refrigeration conditions at temperature $10 \pm 2^{\circ} \mathrm{C}$ and studied for a period of 45 days. At an interval of every 4 days each sample (A, B \& C) was taken and analyzed for ascorbic acid.

1.4.1 Estimation of vitamin - C:-

The procedure for estimation of Vitamin $-\mathrm{C}$ (ascorbic acid AA) was followed as mentioned in AOAC methods 1990 [7].

1.4.2 Statistical analysis:- 
The data was analyzed using appropriate statistical tests one way ANOVA for comparing the average value of the (parameter) ascorbic acid among the treatments. Duncan's multiple range test (DMRT) was used to compare the mean values between pair of treatments.

\section{Results And Discussion}

Vitamin $\mathrm{C}$ is also known as ascorbic acid easily destroyed by oxidation especially at high temperature and easily lost during processing, for preservation. The ascorbic acid content was analyzedfor a period of 45 days at an interval of 4 days. The mean vitamin C content of the sample A, B and C (Table no: 1) was 15.16, and 16.91 and $10.55 \mathrm{mg} / 100 \mathrm{~g}$ of tomato cuts. The vitamin $\mathrm{C}$ content of sample $\mathrm{A}$ and $\mathrm{B}$ was not significantly different. The vitamin $C$ content between sample $A$ and $C$ and sample $B$ and $C$ was significantly different $(\mathrm{P}<0.062, \mathrm{P}<0.4166)$. There was a significant difference between the samples in vitamin $\mathrm{C}$ content (one way ANOVA) ( $\mathrm{F}-5.32, \mathrm{P}=0.047)$.The control sample was deteriorated after $28^{\text {th }}$ day where as the shelf life of sample $\mathrm{A}$ and $\mathrm{B}$ extended up to 44 days.

TABLE 1:- Effect of UV-C and garlic on vitamin C content of tomatoes

\begin{tabular}{|c|l|l|}
\hline \multicolumn{3}{|c|}{ VITAMIN C } \\
\hline S.NO & \multicolumn{1}{|c|}{ SAMPLES } & MEAN \pm Std deviation \\
\hline 1 & A (UV-C Treated) $\mathrm{mg} / 100 \mathrm{~g}$ & $15.16 \pm 2.0209^{\mathrm{a}}$ \\
\hline 2 & B (UV-C + Garlic Treated) $\mathrm{mg} / 100 \mathrm{~g}$ & $16.91 \pm 2.1857^{\mathrm{a}}$ \\
\hline 3 & C (Without Treatment) $\mathrm{mg} / 100 \mathrm{~g}$ & $10.55 \pm 3.0623^{\mathrm{b}}$ \\
\hline
\end{tabular}

*Means having the same super scripts do not differ significantly

Exposing of fresh fruits and vegetables with UV-C is a new approach to extend the storage life of fresh horticulture crops.UV.-C irradiation had no significant effect on citric acid content of treated "Tommy Atkins" mango [8].A reduction in total ascorbic acid content of UV-C irradiated fresh cut mango fruits when compared with control fruits was reported by Gonzalez-Aguilar e tal [9]. In the present study also there is no significant effect of UV-C on the vitamin C content of cut tomatoes.

\section{Conclusion}

Considering the increasing demands of consumers, the use of safe emerging technologies and additives based on natural compounds could be an alternative in the preservation of fresh cut fruits and vegetables. Thus the technologies are applicable to extend the shelf - life of fresh cuts.

\section{References}

[1] Etminan M, et al "The role of tomato products and lycopene in the prevention of prostate cancer"; a Meta analysis of observational studies cancer Epidemiol biomarkers prev. 2004, 13: 340- 345.

[2] Chalmers TC "Effects of ascorbic acid on the common cold. An evaluation of the evidence" Amjmed 1975. April, 58 (4) $532-6$ Review PMID: 1092164.

[3] Han -Yao -Haung et al "Effects of vitamin C on Arthritis and Rheumatism" volume 52, no. 6 June 2005. PP- 1843- 1847

[4] Vicente AR, et al"UV-C treatments reduce decay retain quality and alleviate chilling injury in pepper, post harvest biotech(2005) 35: $69-78$

[5] Davis SRet al"The in vitro susceptibility of scedosporium prolificans to ajoene, allitridium and a raw extract of garlic (Allium sativum). J.Antimicrobial chemother 51 (3) (2003): 5937.

[6] Stevens, C et al.," The germicidal and hermetic effets of UV - C light on reducing brown rot disease and yease micoflora of peaches crop prot. (1998) $17,75-84$

[7] AOAC, 1990- AOAC International official methods of analysis, vol.2 (1990) P. 1058

[8] Gonzalez-Aguilar et al; Use of UV-C irradiation to prevent decay and maintain Postharvest quality of ripe Atkins mangoes. International Journal of Food Science and Technology. 36(2001):767-773

[9] Gonzalez-Aguilar et al 'Improving antioxidant capacity of fresh cut mangoes treated

[10] With UV-C.Journal of Food Science, 72 (2007), S197-S202. 